\title{
Supercharged through a tunnel, is it an action movie? No, it's a novel esophageal reconstruction!
}

\author{
From the Department of Thoracic and Cardiovascular Surgery, Cleveland Clinic, Heart and Vascular Institute, \\ Cleveland, Ohio. \\ Disclosures: Authors have nothing to disclose with regard to commercial support. \\ Received for publication April 17, 2018; accepted for publication April 17, 2018. \\ Address for reprints: Siva Raja, MD, PhD, Cleveland Clinic Foundation, 9500 Euclid Ave J4-1, Cleveland, OH \\ 44195 (E-mail: rajas@ccf.org). \\ J Thorac Cardiovasc Surg 2018;156:867-8 \\ $0022-5223 / \$ 36.00$ \\ Copyright (C) 2018 Published by Elsevier Inc. on behalf of The American Association for Thoracic Surgery \\ https://doi.org/10.1016/j.jtcvs.2018.04.080
}

Rare things are fortunately rare. Dr Yasuda and colleagues ${ }^{1}$ illustrate a novel solution to a, thankfully, rare problem in their case series in this issue of the Journal. In patients with mid or distal esophageal cancer who have no gastric conduit available, and concomitantly, also have a hostile surgical neck, the traditional options for reconstruction are not available. Transmediastinal esophageal replacement, with a posterior mediastinal position proximally, an anterior mediastinal position distally, and a microsurgical vascular anastomosis, has been performed in 4 patients. The novelty of this procedure is that it allows for the avoidance of a difficult surgical neck, and subsequently decreased postoperative swallowing dysfunction or cord injury, all while giving their reconstruction a so-called super-charged second vascular supply. The overall procedure itself seems quite complex. There are 9 steps, 3 intraoperative patient position changes, and the patient needs to have their chest opened and closed twice.

Keeping the complexity of this procedure in mind, the proposed eligibility criteria seem broad. Initially, Dr Yasuda's team ${ }^{1}$ developed the procedure with patients who had esophageal cancer, no stomach, and a hostile neck in mind. However, they also performed their procedure on patients they considered high risk for postoperative aspiration due to age or cerebrovascular disease (who in most cases would receive the traditional neck anastomosis with a substernal colon interposition). It is not entirely clear in the literature that a cervical anastomosis leads to worse swallowing or higher rates of aspiration when compared with an intrathoracic anastomosis. ${ }^{2,3}$ At the very least, it is not clear if any perceived benefit balances out the potential risk of an intrathoracic leak. A recent study ${ }^{4}$ of patients with both intrathoracic and cervical anastomoses that looked at clinical predictors of postoperative aspiration after esophagectomy only found operative time to be associated with a higher rate of postoperative aspiration.

For the patients with truly no possibility for cervical anastomosis and esophageal cancer with no stomach, the

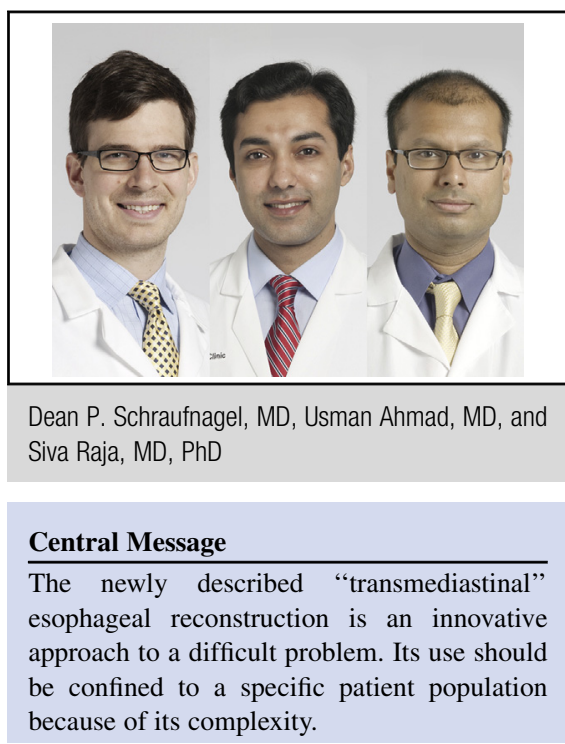

See Article page 859. transmediastinal esophageal replacement technique is another tool in the tool box. This work falls in line with the idea that in surgery, difficult problems help drive the development of novel therapies. These novel therapies require one to draw upon the art of making high-risk, high reward decisions in well-informed patients willing to take those risks. ${ }^{5}$

For such patients with esophageal cancer, no stomach, and extensive prior surgery or radiation in the neck, Dr Yasuda and colleagues ${ }^{1}$ have described an innovative solution to an otherwise impossible problem. However, with success in this select patient population, caution should be taken before expanding its indications to include those with high risk for postoperative aspiration or to use in lessspecialized centers. When we get a new shiny hammer, sometimes, everything starts to look like a nail. Although supercharging and going through a tunnel may be exciting like an action movie, patients may not always benefit from that much action if a simpler road is the safer route.

\section{References}

1. Yasuda T, Shiraishi O, Iwama M, Makino T, Kato H, Kimura Y. Novel esophageal reconstruction technique via trans-mediastinal route from posterior to anterior mediastinum after esophagectomy. J Thorac Cardiovasc Surg. 2018; 156:859-66. 
2. Wormald JCR, Bennett J, van Leuven M, Lewis MPN. Does the site of anastomosis for esophagectomy affect long-term quality of life? Dis Esophagus. 2016;29:93-8.

3. Irino T, Tsekrekos A, Coppola A, Scandavini CM, Shetye A, Lundell L, et al. Longterm functional outcomes after replacement of the esophagus with gastric, colonic, or jejunal conduits: a systematic literature review. Dis Esophagus. 2017;30:1-11.
4. Lee SY, Cheon HJ, Kim SJ, Shim YM, Zo JI, Hwang JH. Clinical predictors of aspiration after esophagectomy in esophageal cancer patients. Support Care Cancer. 2016;24:295-9.

5. Bribriesco A, Ahmad U, Raja S. The price of innovation: a primer on high risk, high reward surgery. J Thorac Dis. 2017;9:4323-4. 